Conference paper

\title{
Single Ion Implantation with Scanning Probe Alignment
}

\author{
A. Persaud, F. I. Allen, T. Schenkel ${ }^{1}$, I. W. Rangelow*, F. Gicquel, K. Ivanova*, S. J. \\ Park , J. A. Liddle, and J. Bokor \\ E. O. Lawrence Berkeley National Laboratory, Berkeley, CA \\ *Institute of Microstructure Technologies and Analytics, University of Kassel, Germany
}

We present results from our development of a single ion implantation technique integrated with a scanning force microscope. Accurate alignment at the $5 \mathrm{~nm}$ level is a crucial requirement for reliable single ion placement. We address this through integration of the ion beam with a scanning probe tip containing an aperture. Single ion registration is based on detection of secondary electron bursts from single, high charge state ions. We describe formation of scanning probe tips with holes and sensing poles by focused ion and electron beam processing (drilling and thin film deposition). Ion transport studies through apertures show stable transmission for $>10 \mathrm{~h}$ with $1 \mathrm{nA}$ scale beam intensities on pre-collimators. 


\section{Introduction}

Single ion implantation is a "top down" path to the formation of single atom devices [1-3]. An example of proposed single atom devices are impurity spin based quantum computers were information is encoded in the electron and nuclear spins of individual atoms in arrays aligned to control gates and readout Single Electron Transistor (SET) structures [4]. In this article we present results from our development of a single ion implantation technique integrated with a scanning force microscope $[5,6]$. Accurate alignment at the $5-10 \mathrm{~nm}$ level is a crucial requirement for reliable single ion placement. Accuracy requirements are specific to quantum computer schemes and two qubit interactions employed. Electron spin coupling by exchange interaction requires a donor atom spacing of 10 to $30 \mathrm{~nm}$, while "electron shuttling" would allow spacings of $>100 \mathrm{~nm}[4,8]$. The placement accuracy should be a small fraction of the qubit spacing, and ions should be placed with close to $100 \%$ efficiency. The state of the art in single ion placement is an aiming accuracy of $60 \mathrm{~nm}$ and a detection efficiency of $90 \%$

for $60 \mathrm{keV} \mathrm{Si}^{2+}$ ions by Shinnada et al. [9]. Yang et al. have achieved an impressive detection efficiency of $85 \%$ for $14 \mathrm{keV} \mathrm{P^{+ }}$ ions in a solid state detector adaptation with $20 \mathrm{~nm}$ holes in resist [3]. Here, no alignment procedure was implemented and the two positions were implanted at random until two ions had been registered.

We address the alignment problem through integration of the ion beam with a scanning probe tip containing an aperture $[5,6]$. Integration of the ion beam with a scanning probe is attractive, because it allows non-destructive imaging of sample features with nm scale resolution.

\footnotetext{
${ }^{1}$ Email: T_Schenkel@LBL.gov, phone: 510-486-6674
} 


\section{Instrument design and tip formation}

Figure 1 shows the conceptual layout of our approach. The Scanning Force Microscope with piezoresistive sensors [10] images the device region to be implanted in a high vacuum chamber (base pressure $10^{-8}$ torr). Following imaging, sites for implantation are selected, and ions are allowed to reach the probe tip. The probe tip is pierced with a hole, allowing it to transmit ions at a rate of a few Hz. Secondary electron bursts from the impact of individual highly charged dopant ions (e. g. ${ }^{31} \mathrm{P}^{13+}$ ) are detected in a scintillator coupled to a photomultiplier tube. Pulse heights from detection of multiple electrons from one highly charged ion impact are well separated from single electron background events $[1,5,11]$, and low multiplicity events from ion hits on apertures. Secondary electrons are guided to the detector with electrical and magnetic fields, so that the probe tip can be held in close proximity $(<1 \mu \mathrm{m})$ to the sample. Following each ion impact, the beam is blanked within a few $\mu \mathrm{s}$, and the process of imaging and alignment is repeated. Ion arrival times at the tip are random, but the transmission rate is small enough to ensure that only one ion impinges on a designated area before the beam is blocked.

The diameter of the hole in the scanning probe tip limits the minimum achievable placement accuracy, and we have developed a technique for nm-scale hole formation [7]. Cantilevers (Figure 2 a) with piezoresistive sensors [12] are mounted on $\mathrm{Si}_{3} \mathrm{~N}_{4}$ membranes. Using a low divergence, highly focused ion beam in a conventional FIB, we drill $\sim 1 \mu \mathrm{m}$ size holes through both the tip and the membrane. The distance between cantilevers and membranes is $\sim 0.35 \mathrm{~mm}$, and the hole in the membrane is enlarged by a factor of two compared to the hole in the cantilever (Fig. 2 b) due broadening of the FIB beam past the focal plane. 
The hole in the tip is then reduced in diameter by local, electron and ion beam assisted deposition of $\mathrm{Pt}$ (or TEOS oxide). The hole in the membrane is aligned to the hole in the tip and pre-collimates the implant beam. In Figure 2 c), we show the original hole from Figure 2 b) after hole closing by ion beam assisted Pt deposition. A sharp pole with a radius of curvature of about $30 \mathrm{~nm}$ was then grown next to the residual hole. Calibration of the distance of the imaging surface to the hole transmission aperture is crucial for accurate ion placement.

In Figure 3, we show data from ion transmission measurements through aligned holes in cantilever and membrane with a relatively large tip opening of $1.5 \mu \mathrm{m}$. As expected, very little charge exchange was observed [1], and ion transmission rates were stable for $>10 \mathrm{~h}$ with beam currents of $1 \mathrm{nA}$ exposing the pre-collimating membrane ( $0.5 \mathrm{~mm}$ diameter). Similar results were obtained again with the same cantilever-membrane sample after the hole diameter was reduced to $200 \mathrm{~nm}$ in a later FIB session. Use of a pre-collimating aperture limits the effect of hole closing, which was reported for high currant exposures large areas surrounding small holes in silicon nitride membranes [13].

\section{Outlook}

Single ion implantation with scanning probe alignment promises single ion placement with high resolution and efficiency. Several issues are being addressed in ongoing studies. E. g., calibration of imaging surfaces with respect to beam limiting holes will be crucial for achieving $<10 \mathrm{~nm}$ placement accuracies. FIB processing allows formation of cantilevers with nanometer scale holes and sharp imaging poles. Prolonged ion beam transport is possible through small holes with pre-collimating apertures avoiding hole closing by ad-atom diffusion. 


\section{Acknowledgments}

We thank F. Ogletree for stimulating discussions and support with the AFM configuration, and the staff of the UC Berkeley Microlab, and the National Center for Electron Microscopy for their technical support. This work was supported by NSA and ARDA under ARO contract number MOD707501, and by the U. S. DOE under contract No. DE-AC0376SF00098.

\section{References}

[1] T. Schenkel, et al., J. Appl. Phys. 94, 7017 (2003)

[2] R. G. Clark et al., Phil. Trans. Roy. Soc. Lond. A 361, 1451 (2003)

[3] C. Yang, D. N. Jamieson, C. Pakes, S. Prawer, A. Dzurak, F. Stanley, P. Spizziri, L.

Macks, E.Gauja, R. G. Clark. J. J. Appl. Phys. 42, 4124 (2003)

[4] B. E. Kane, Nature 393, 133 (1998); R. de Sousa et al., cond-mat/0311403

[5] S.-J. Park, A. Persaud, J. A. Liddle, J. Nilsson, J. Bokor, D. H. Schneider, I. Rangelow and T. Schenkel, Microelectr. Engin. 73-74, 695 (2004)

[6] T. Schenkel, I. W. Rangelow, R. Keller, S. J. Park, J. Nilsson, A. Persaud, V. R.

Radmilovic, P. Grabiec, D. H. Schneider, J. A. Liddle, and J. Bokor, Nucl. Instr. Meth. B 219$220,200(2004)$

[7] T. Schenkel, V. Radmilovic, E.A. Stach, S.-J. Park and A. Persaud, J. Vac. Sci. Technol. B $21,2720(2003)$

[8] A. J. Skinner et al., Phys. Rev. Lett. 90, 087901 (2003)

[9] T. Shinnada, H. Koyama, C. Hinoshita, K. Imamura, I. Ohdomari, Jap. J. Appl. Phys. 41, L287 (2002)

[10] I. W. Rangelow, at al., SPIE Vol. 2879, (1996) pp. 56-65 
[11] T. Schenkel et al., Journal of Quantum Information Processing, Special Issue on Quantum Computer Development, in press

[12] P. Grabiec, J. Radojewski, M. Zaborowski, K. Domanski, T. Schenkel, and I. W.

Rangelow, J. Vac. Sci. Tech. B 22, 16 (2004); R. Pedrak, Tzv. Ivanov, K. Ivanova, T.

Gotszalk, N. Abedinov, and I. W. Rangelow, K. Edinger, E. Tomerov, T. Schenkel, and P.

Hudek, J. Vac. Sci. Technol. B 21, 3102 (2003)

[13] J. Li, et al., Nature 412, 166 (2002) 
Figure 1: Schematic illustration of the Single Ion Implantation setup with scanning probe alignment alignment. Piezo-resistive cantilever with tip (1) and small aperture (2), mounted on a pre-collimation membrane (3); and secondary electron detector (4). The sample is a set of $\mathrm{Cu}$ lines ( $2 \mu \mathrm{m}$ wide, $\sim 40 \mathrm{~nm}$ high on $\mathrm{Si}$ ) shown in a scanning force microscope image collected in contact mode with piezoresistive sensors at a vacuum of $2 \times 10^{-7}$ torr in the ion beam line. Dashed lines indicate trajectories of secondary electrons in electrical and magnetic guiding fields.

Figure 2: a) Scanning probe cantilever mounted over a silicon nitride membrane. b) A hole is drilled though both the cantilever and the membrane. c) The hole in the cantilever is closed by ion and electron beam assisted Pt deposition, and an imaging pole is growth next to the hole also by ion and electron beam assisted Pt deposition.

Figure 3: Results from charge state analysis of $\mathrm{Ar}^{2+}$ ions $(\mathrm{E}=7.2 \mathrm{keV})$ transmitted through aligned holes in tip and membrane with a tip opening of $1.5 \mu \mathrm{m}$. Over $99 \%$ of ions transmit the tip without charge exchange, and for extended exposures ( $1 \mathrm{nA}$ on the pre-collimating membrane for $>10 \mathrm{~h}$ ). 


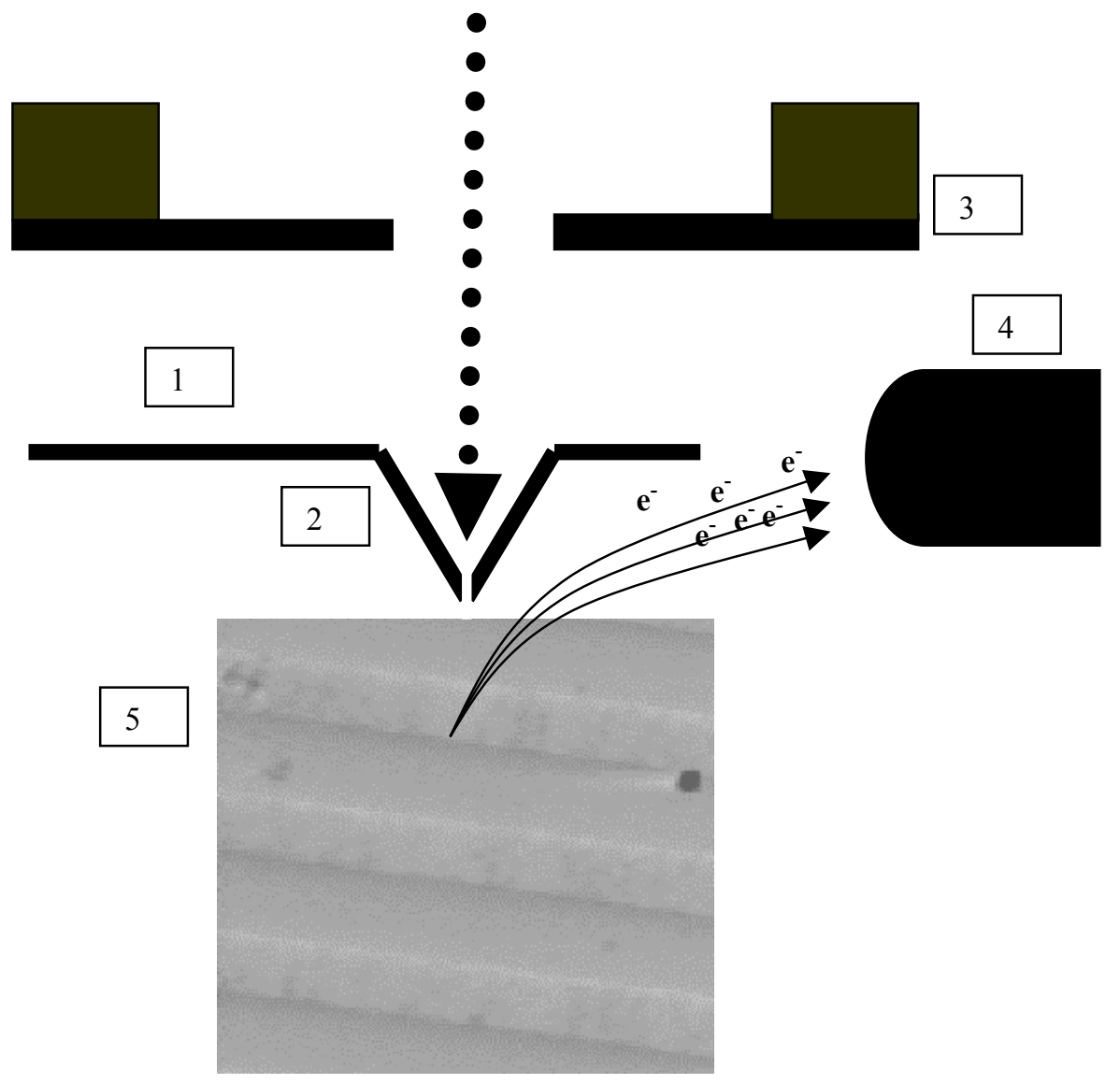




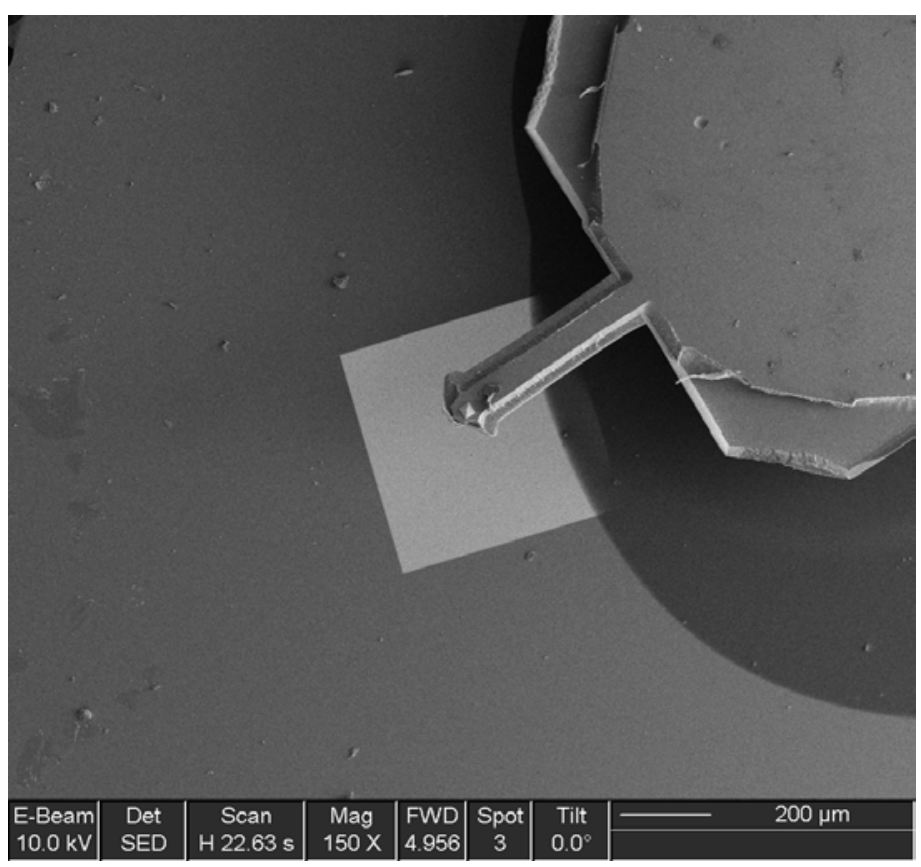




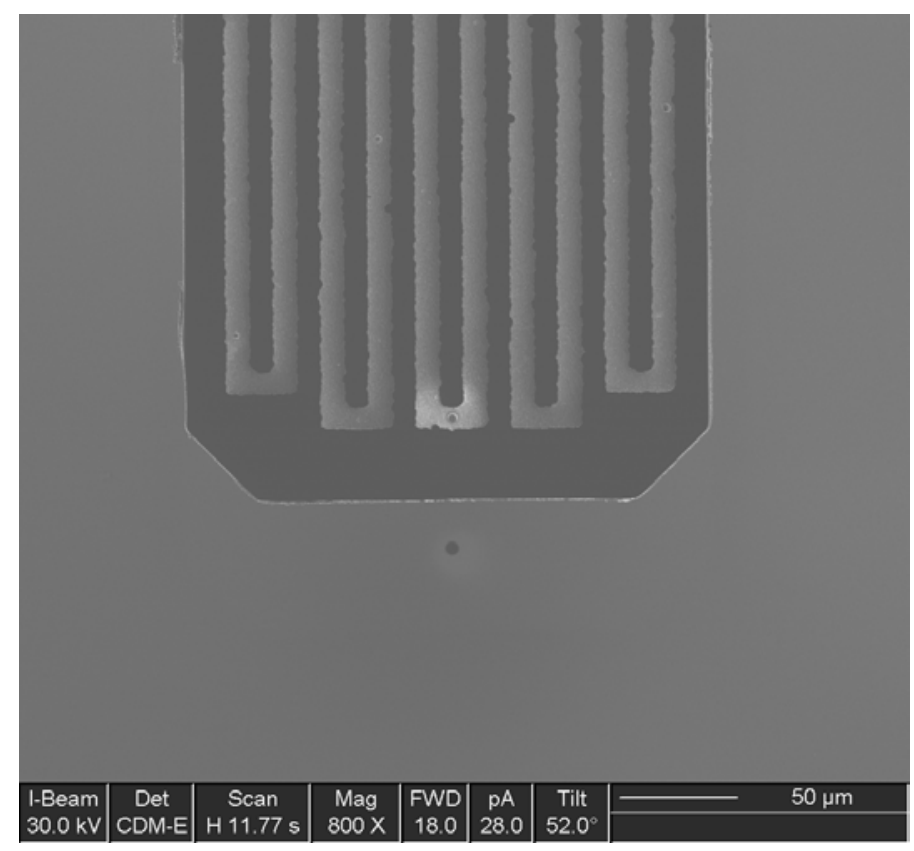




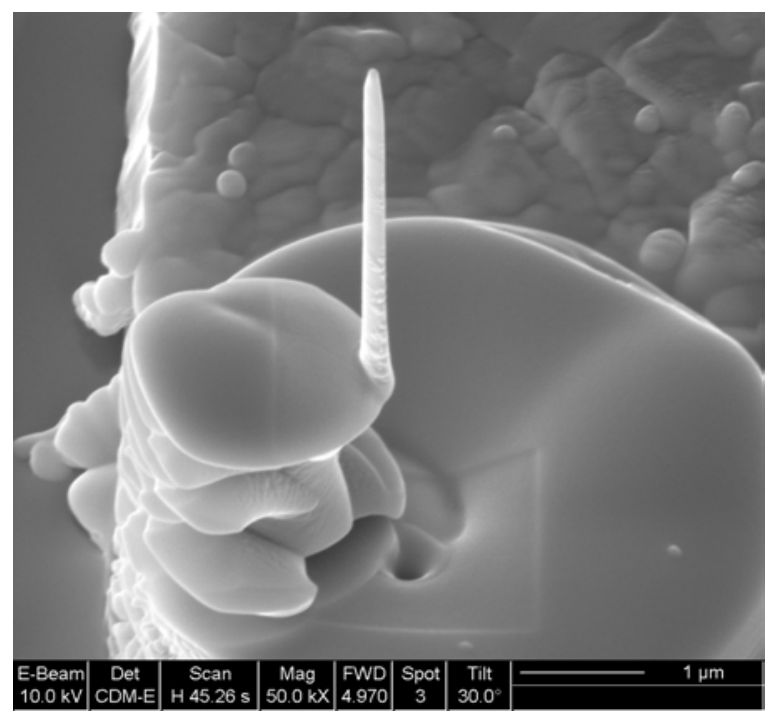




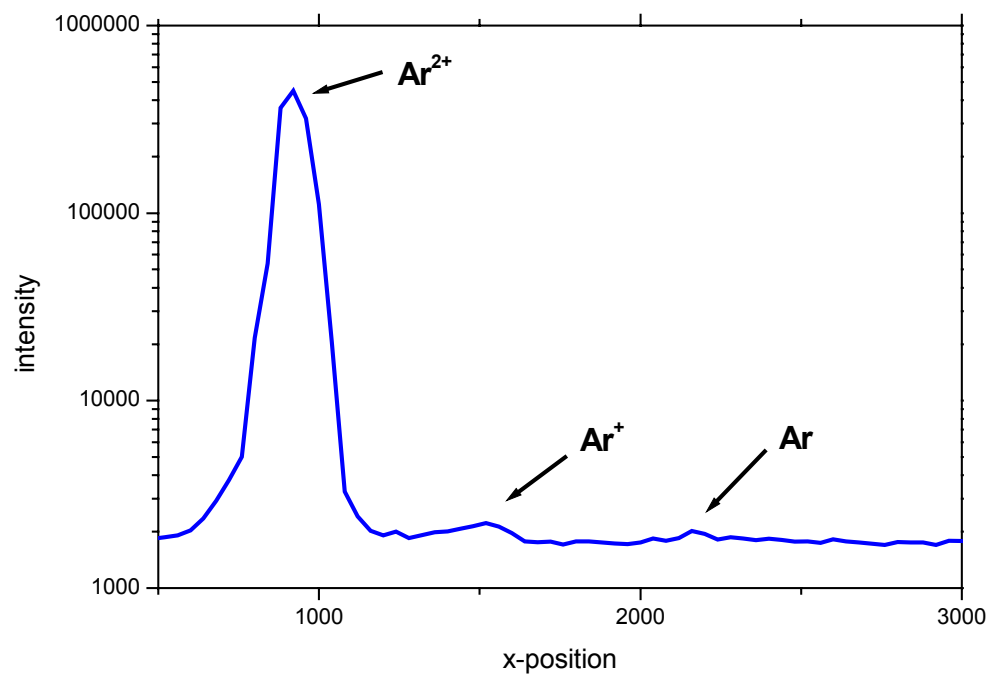

